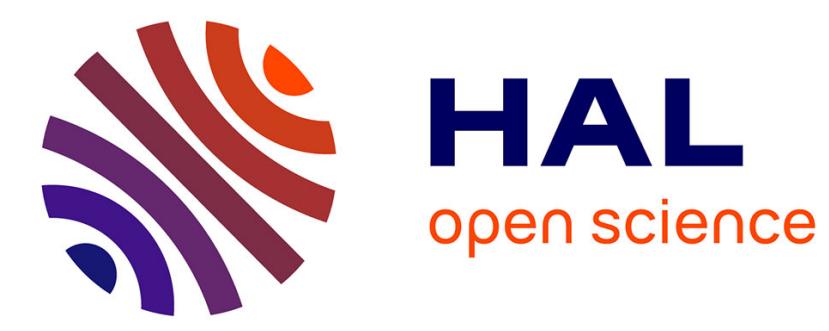

\title{
On the static and dynamic effects of sensitization of emission in laser crystals
}

\author{
V. Lupei, A. Lupei, G. Boulon
}

\section{To cite this version:}

V. Lupei, A. Lupei, G. Boulon. On the static and dynamic effects of sensitization of emission in laser crystals. Journal de Physique IV Proceedings, 1994, 04 (C4), pp.C4-305-C4-310. 10.1051/jp4:1994475 . jpa-00252738

\section{HAL Id: jpa-00252738 https://hal.science/jpa-00252738}

Submitted on 1 Jan 1994

HAL is a multi-disciplinary open access archive for the deposit and dissemination of scientific research documents, whether they are published or not. The documents may come from teaching and research institutions in France or abroad, or from public or private research centers.
L'archive ouverte pluridisciplinaire HAL, est destinée au dépôt et à la diffusion de documents scientifiques de niveau recherche, publiés ou non, émanant des établissements d'enseignement et de recherche français ou étrangers, des laboratoires publics ou privés. 


\title{
On the static and dynamic effects of sensitization of emission in laser crystals
}

\author{
V. LUPEI, A. LUPEI and G. BOULON*
}

Institute of Atomic Physics, 76900 Bucharest, Romania

* Université Claude Bernard Lyon I, LPCML, URA 442 du CNRS, 69622 Villeurbanne, France

Abstract. It is shown that the mutual static crystal fieid perturbation produced by the sensitizer and activator ions at each other's site could nociti joth the radiative and energy transfer characteristics for near sensitizer-activator pairs in co-joped crgstals. These effects nodify the spectral and tempora! characteristics of the activator emission in the sensitized systems. The implication of thess static effects on the estimation of sensitizer-activator transfer efficiency by using the iuninescence decay of the conors is also jiscussed.

Sensitization of weakly absorbing rare-earth laser active ions by co-doping the crystals with transition ions that match better the emission of pump light sources is regarded as an important means to improve the efficiency of solid state lasers. An essential condition for sensitization is the existence of a fast transfer of excitation from sensitizer (donor-D) to the activator (acceptor $\mathrm{A}$ ). The energy transfer could be direct or it could be assisted by migration on the donor system; in this work we shall refer exclusively to the first situation.

Usually the donor and acceptor systems are considered as homogeneous, i.e. all the members of the system have identical spectral and temporal properties of absorption and emission. The characteristics of the energy transfer could be determined from the investigation of the populations of the donor and acceptor energy levels involved in transfer, by monitoring the temporal characteristics of luminescence and comparing with the situation of simply (only donor or only acceptor) - doped crystals.

Thus, the emission of a homogeneous system of donors, which in absence of acceptors shows an exponential decay with lifetime $\tau_{D}$ is modified by the transfer to the acceptors to:

$$
I\left(t, C_{A}\right)=I(0) \exp \left(-\frac{t}{\tau_{D}}\right) \exp \left[-P\left(t, C_{A}\right)\right]
$$

where $C$ is the relative concentration of acceptors and $P(t, C)$ is the acceptor ensemble-averaged probability that the donor is not desexcited at the time $t$ by energy transfer to an acceptor placed at any point in the crystal. For these homogeneous donor systems, the relative quantum efficiency of emission is given by:

$$
\eta_{I}\left(C_{A}\right)=\frac{1}{\tau_{D}} \int \frac{I\left(C_{A}, t\right)}{I(0)} d t
$$

and that of the energy transfer is:

$$
\eta_{t x}\left(C_{A}\right)=1-\eta_{I}\left(C_{A}\right)
$$

The energy transfer leads to the population of the acceptor level when pumping in the donor absorption bands and, assuming that in absence of the donor it decays exponentially with lifetime $\tau_{a}$, the acceptor population $n_{a}$ is given by: 


$$
n_{A}(t)=n_{D}(0) \exp \left(-\frac{t}{\tau_{A}}\right) \int_{0}^{1} \exp \left[\left(\frac{1}{\tau_{A}}-\frac{1}{\tau_{D}}\right) q\right] \exp [-P(q)] \frac{d P(q)}{d q} d q
$$

The transfer function $P(t)$ could be calculated for a given model of distribution of acceptors in crystal provided the transfer rate from donor to any acceptor $i$ at the distance $r_{i}$ is known. This rate depends on the microparameter of interaction $\mathrm{C}_{\text {in }}$ determined essentially by the superposition integral of donor emission and acceptor absorption as well as on the distance $r_{1}$, the last dependence being determined by the nature of $\mathrm{D}-\mathrm{A}$ interaction responsible for transfer. For multipolar interactions the rate of transfer could be written as:

$$
W_{M P}=W_{d-d}+W_{d-q}+W_{p-q}
$$

where $d$ stands for dipole and $q$ for quadrupole; usually for the distant $D-A$ pairs the $d-d$ interaction dominates, but for near pairs a situation of multiple interaction involving several types of interactions could hold. For multipolar interactions the rate of transfer depends on $r_{i}$ as [1]:

$$
W_{i}=\frac{C_{D A}}{r_{1}^{s}}
$$

with $s=6$ for $d-d, 8$ for $d-q$ and 10 for $d-d$ interactions. It is seen that the $d-d$ interaction which usually governs the transfer gives the lowest dependence on $D-A$ distance.

The superexchange interaction shows a stronger exponential dependence on distance. Thus in case of the homogeneous systems of donors the rate of $D-A$ transfer depends (besides of microparameter $C_{r,}$ ) only on the $D-A$ distance.

Once the rates $W$ are known the averaging depends on the model of acceptor distribution. Thus, in case of a continuous and uniform distribution of acceptors, we have [2]:

$$
P(t)=\gamma t^{3 / s}=4 \pi n_{A} \Gamma\left(1-\frac{3}{s}\right) C_{D A}^{3 / s} t^{3 / s}
$$

and the parameters $\gamma$ (or $C_{\text {i }}$ ) and $s$ could be simply determined from the fit of eq. (7) to the experimental data. This equation does not give usually a correct description of the beginning of luminescence decay because the continuous uniform model assumes a finite probability of finding the acceptor at the donor site. This shortcoming could be avoided by assuming a discrete and random distribution of the acceptor at the lattice site available for them, with a probability equal with $C_{1}$ (regardless of site). In this case the transfer function is [3]:

$$
P(t)=-\sum_{i} \ln \left[1-C_{A}+C_{A} \exp \left(-W_{i} t\right)\right]
$$

and it gives a correct description of the decay at any time $t$. At early times the function $P(t)$ could be approximated by a linear function of time, while at long times it could be approximated by eq. (7) plus a shift constant.

If for the first $j$ sites the transfer rates are very large so as $\exp \left(-\mathrm{W}_{i} t\right) \sim 0$ even at very short times $t[4,5]$ : 


$$
P(t) \propto \sum_{i=1}^{j} \ln \left(1-C_{A}\right)-\sum_{i>j} \ln \left[1-C_{A}+C_{A} \exp \left(-W_{i} t\right)\right]
$$

which for low acceptor concentrations $C_{A}$ becomes:

$$
P(t) \propto-j C_{A}-\sum_{i>f} \ln \left[1-C_{A}+C_{A} \exp \left(-W_{i} t\right)\right]
$$

The first term in the right side of eq. (9) and (10) is independent of time and so it predicts a sudden drop of emission at or near $t=0$. This drop is usually not seen experimentally and it corresponds in fact to an almost complete quenching of the donor emission in the near $D-A$ pairs due to a very fast excitation transfer to acceptor. What is really seen in the decay in this case is the effect of the second term in (10); however the sum over $j$ is now truncated with the exclusion of the first $j$ sites and this should be taken into account for a correct description of decay. Because of the first term in (10) the normalized decay I/I should not start from the value 1 at $t=0$ but from $(1-j C)$. The situation could be even more complex when besides these $j$ sites of very fast transfer there are also several sites with stronger interaction (due possibly to a multiple interaction situation) than the distant sites. An accurate analysis of the decay in all these cases is crucial for a correct estimation of quantum efficiencies (2) or (3).

Recent high resolution spectroscopic studies on sensitized crystals have shown [6-11] that the sensitizers and the activators could produce strong perturbation of the crystal field it each other's site. These perturbations could depend not only on the donor-acceptor distance but also on the direction of perturbation with respect to the local symmetry axes of the site. The static perturbations transform the donor and acceptor in non-homogeneous systems, composed of sub-systems each of them characterized by a given static perturbation.

The main static effects of these crystal field perturbation are:

(i) the shift of energy levels manifested by the apparition of sateliites in absorption and emission. Such satellites have been observed in garnets activated with $\mathrm{Nd}$ and sensitized with $\mathrm{Cr}$ [6] or activated with $\mathrm{Tm}$ and sensitized with $\mathrm{Cr}[7,8,9]$ or $\mathrm{Fe}[10,11]$ and in many other systems. The satellite structure depends on the nature and ionic size of the sensitizer and acceptor ions and on the crystalline structure of the host crystal. Because of the angular dependence of the perturbation effect with respect to the local axes of symmetry (especially when this is low), in many instances perturbing ions placed in the various sites of a given coordination sphere could produce different crystal field perturbations at the central ion site, resulting in several satellites, although the $D-A$ distances are identical.

(ii) modification of the selection rules for optical transitions due to a lowering of symmetry. Several important cases could be illustrative:

- when the local symmetry of the site available to the sensitizer has inversion symmetry, the electric dipole transitions between the state belonging to a given electronic configurations are parity forbidden. The perturbation produced by an activator ion placed at an adjacent lattice site could lower the local symmetry at the sensitizer site removing the center of symmetry and the electric dipole transitions become allowed due to the mixture of states from configurations with different parity:

- in case of non-Kramers rare-earth activators (such as $\mathrm{Tm}^{3+}, \mathrm{Ho}^{3+}, \mathrm{Pr}^{3+}$

and so on) the local symmetry $D_{2}$ of the dodecahedral site in garnet lattices, is orthorhombic, and the transitions between the crystal field components labelled by an identical irreducible representation of this group 
are forbidden: they represent usually over $25 \%$ of the possible transitions. However, for all the point symetry groups lower that $D_{2}$ these transitions are allowed. The sensitizer ions in garnet crystals could occupy the octahedral or/and tetrahedral sites and regardless of the site occupied near the activator, the perturbation produced at its site lowers the local symmetry to a subgroup $D_{3}$. Because of this absorption or emission spectra of the nonKramers rare-earth activators in perturbed sites in garnets are richer than those of similar ions in non-perturbed sites, as seen in YAG:Tm sensitized by $\mathrm{Cr}$ or $\mathrm{Fe}$. We must mention that a similar effect could be produced due to the presence of a lattice deffect in the vicinity of the activator.

(iii) modification of the transition probabilities, especially for the non-Kramers ions. This could lead to an enhanced spontaneous emission coefficient for emitters in perturbed sites, accompanied by a corresponding reduction of the radiative lifetimes.

These static perturbation effects could influence the properties of the near sensitizer- activator pairs by:

A. Modification of the absorption properties (spectral distribution, line intensities); because of this the excitation of the sensitizer by the pump radiation could be different in perturbed and non-perturbed sites.

$B$. They could modify the $D-A$ energy transfer owing to a selective modification of the superposition integral of donor emission and acceptor absorption. In view of the particularities of the static perturbations discussed above this could induce a radial and angular dependence of the microparameter:

$$
C_{D A} \equiv C_{D A i} \equiv C_{D A}\left(r_{i}, \theta_{i}, \varphi_{i}\right)
$$

where the polar angles $\theta_{\text {; }}$ and $\varphi$ define the position of the perturbing ion with respect to the local symmetry axes.

In this case the transfer rate might not only depend on the angular position of the perturbator:

$$
W_{i}=W\left(r_{i}, \theta_{i}, \varphi_{i}\right)
$$

but its radial dependence could be different from that given by (6), without even changing the nature of $\mathrm{D}-\mathrm{A}$ interaction. An enhancement of the transfer rate could be thus obtained, but the new radial dependence, that includes both the dependencies (6) and (11) will be not described by a clear mathematical law. This effect could be important for the near D-A pairs, where a multiple interaction situation could be also hold, making the analysis very difficult.

c. Modification of the emission properties of the activator:

- the spectral composition of the activator emission could be changed due to the shift of the spectral lines for perturbed sites as well as due to the apparition in emission of lines which are forbidden for the non-perturbed sites;

- the intensity of emission could be modified due to the change of the spontaneous emission coefficient as well as due to a selective excitation of the activator sites for which the $D-A$ energy transfer is faster;

- the temporal behaviour of emission could be modified by several processes: a different rate of excitation of various activator sites due to large differences in the $D-A$ transfer rates, a selective modification of the radiative lifetime for the perturbed activator sites and an induced selectively of the non-radiative process (e.q. energy transfer processes inside the system of activators) for these sites.

Thus by a complex correlation of the static perturbation processes produced by the co-doping of the laser crystals with activator and sensitizer ions, with the dynamic characteristics of excitation of the activator by 
energy transfer, the spectral and temporal emission properties of the activators in the sensitized crystals could be strongly different from those corresponding to the non-sensitized crystals. This effect is clearly seen in the $\mathrm{H}_{4} \rightarrow \mathrm{H}_{\text {e }}$ emission of $\mathrm{Tm}^{3+}$ in garnet crystals sensitized by $\mathrm{Cr}^{3+}[7,8,9]$ in octahedral sites or $\mathrm{Fe}^{3 t}$ in tetrahedral sites $[10,11]$; at high activator concentrations, the emission at early times after a short excitation pulse in the sensitizer absorption bands is dominated by the new satellites induced by this co-doping, and the emission has different spectral and temporal behaviour from that of the less or non-perturbed sites.

These modifications influence strongly the estimations of transfer efficiency from the luminescence decay data. Thus, if due to the static perturbations produced by the near activators, the sensitizer ions could be classified in $\mathrm{k}$ subsystems, each of them containing a density of ions $n_{k}$ emitting at a wavelength $\lambda_{k}$ and having the spontaneous emission coefficient modified from $A$ ( the value for distant $D-A$ pairs) to $A$ and the radiative lifetime changed from $\tau_{n}$ to $\tau_{b}$, the total emission intensity of the system of donors after a short pulse excitation is a sum of the contributions of all the subsystems:

$$
I(t)=\sum_{k} I_{k}(t)=\sum_{k} A_{k} n_{k}(t)
$$

The luminescence decay for each subsystem is:

$$
I_{k}(t)=A_{k} n_{k}(0) \exp \left(-\frac{t}{\tau_{k}}\right) \exp \left[-P_{k}(t)\right]
$$

where the energy transfer function is:

$$
P_{k}(t)=\sum_{i(k)} \ln \left[1-C_{A}+C_{A} \exp \left(W_{i} t\right)\right]
$$

If we denote:

$$
\alpha_{k}=\frac{A_{k}}{A} \text {, and } \rightarrow \beta_{k}=\exp \left[-\left(\frac{t}{\tau_{k}}-\frac{t}{\tau_{D}}\right)\right]
$$

and assume that the static perturbation modifies only the radiative properties of the perturbed centers, i.e.

$$
\boldsymbol{\beta}_{k}=\exp \left[-A\left(\boldsymbol{\alpha}_{k}-1\right) t\right]
$$

the total emission from the donor centers becomes:

$$
I(t)=A \exp \left(-\frac{t}{\tau_{D}}\right) \sum_{k} \alpha_{k} n_{k}(0)\left[-A\left(\alpha_{k}-1\right) t\right] \exp \left[-P_{k}(t)\right]
$$

Eq. (18) shows that the enhancement of the spontaneous emission coefficient together with the corresponding reduction of the radiative lifetime, coupled with a faster energy transfer to the nearest activator sites could modify the temporal behaviour of the donor emission leading to a fast drop at the beginning of decay, followed by a slower part corresponding to the transfer to distant acceptor ions. Due to the truncation of the lattice sum with exclusion of the first acceptor sites which determine the peculiar behaviour of the beginning of decay, the other part is apparently slower than a normal decay. In many cases due to the very fast transfer to several of the 
nearest acceptors ions, the sudden drop predicted by eq (10) could take place. A very important aspect of the static perturbative effect is that the emission intensity given by eq(18) is not simply proportional with the total donor population $n_{n}=\Sigma n_{1}$ as in case of the homogeneous systems ( $I=A n$ ) but with the sum of products $A, n$, which reflect not only the effect of energy transfer on the donor population but also the change of the radiative properties due to perturbation. Because of this the temporal evolution of donor emission intensity could no longer be used for calculation of emission quantum efficiency by eq. (2) or (3). This shows that the evolution of population $n,(t)$ for each subsystem should be extracted from observed emission decay and then the sum $\Sigma n_{\text {. }}(t)$ could be used for estimation of transfer efficiency.

The effect of the mutual static perturbation of sensitizer and activator ion on the emission of acceptors could be treated in a similar way: the system of activator ions becomes inhomogeneous and it could be considered as composed of m subsystems, according to the perturbation produced by the sensitizer ions at the activator ion site. We mention that generally the number $m$ could be different from the number $k$ defined earlier although a correspondence between the subsystems of sensitizers and activators could be established in each particular case.

In conclusion these studies show that due to the effect of mutual static crystal field perturbations of the sensitizer and activator ions on their absorbtion and emission properties and on the $D-A$ energy transfer, the spectral composition, the intensity and the temporal behaviour of the sensitized emission could be different from emission of the active system without sensitizer. It also shows that a detailed and accurate analysis of the modification of donor radiative properties and of the selective aspects of the energy transfer for the near D-A pairs is necessary in order to get a correct estimation of the global D-A energy transfer efficiency from the temporal behaviour of donor emission.

\section{References:}

1. D.I.Dexter, J.Chem.Phys. 21, 836 (1953).

2. M.Inokuti, F.Hirayama, J.Chem.Phys. 43, 1978 (1965).

3. I.S.Golubov, Yu.V.Konobeev, Sov.Phys.Sol.St. 13, 2679 (1972).

4. V.Lupei, A.Lupei, S.Georgescu, W.M.Yen, J.App1.PhYs. 66, 378 (1989).

5. V.Lupei, A.Lupei, S.Georgescu, I.Ursu, Appl.Phys.Lett. 59, 905 (1991).

6. J.A.Mares, W.Nie, G.Boulon, J.de Phys. 51, 1655 (1990).

7. W.Nie, Y.Kalinsky, C.Pedrini, A.Montiel, G.Boulon, Opt. Quant.Electron, $22, \mathrm{~S}-123$ (1990).

8. V.Lupei, L.Lou, G.Boulon,A.Lupei, C.Tiseanu, J.de Physique I3,1245 (1993).

9. A.Brenier, G.Boulon, C.Pedrini, C.Madej, Opt.Mat. 1, 299 (1992).

10. A.Brenier, G.Boulon, C.Pedrini, C.Madej, M.J.Elejalde, A.Lupei, C.Tiseanu, S.Georgescu, Proc.SPIE, Sol.St.Lasers IV (1993).

11. V.Lupei, G.Boulon, A.Lupei, M.J.Elejalde, A.Brenier, C.Pedrini, Phys, Rev.B (in print). 